\title{
Genome-wide prediction of transcription start site in four conifer species
}

\author{
Eugeniia I. Bondar \\ Laboratory of Forest Genomics \\ Siberian Federal University \\ Krasnoyarsk, Russia \\ Laboratory of Genomeic Research and \\ Biotechnology \\ FRC KSC SB RAS \\ Krasnoyarsk, Russia \\ bondar.ev@ksc.krasn.ru \\ Vadim V. Sharov \\ Laboratory of Genomeic Research and \\ Biotechnology \\ FRC KSC SB RAS \\ Krasnoyarsk, Russia \\ Laboratory of Forest Genomics \\ Siberian Federal University \\ Krasnoyarsk, Russia \\ Department of High Performance \\ Computing \\ Siberian Federal University \\ Krasnoyarsk, Russia \\ sharvadim07@ya.ru
}

\author{
Dmitry A. Kuzmin \\ Laboratory of Forest Genomics \\ Siberian Federal University \\ Krasnoyarsk, Russia \\ Department of High Performance \\ Computing \\ Siberian Federal University \\ Krasnoyarsk, Russia \\ dm.kuzmin@gmail.com \\ Tatiana V. Tatarinova \\ Department of Biology \\ University of La Verne \\ La Verne, USA \\ Functional Genomics Group \\ Vavilov Institute for General Genetics \\ Moscow, Russia \\ Siberian Federal University \\ Krasnoyarsk, Russia \\ Bioinformatics Center of IITP RAS \\ Moscow, Russia \\ ttatarinova@laverne.edu
}

\author{
Konstantin V. Krutovsky \\ Laboratory of Forest Genomics \\ Siberian Federal University \\ Krasnoyarsk, Russia \\ Department of Forest Genetics and \\ Forest Tree Breeding \\ Georg-August University of Göttingen \\ Göttingen, Germany \\ Laboratory of Population Genetics \\ Vavilov Institute of General Genetics \\ Moscow, Russia \\ Department of Ecosystem Science and \\ Management \\ Texas A\&M University \\ College Station, TX, USA \\ konstantin.krutovsky@forst.uni- \\ goettingen.de
}

\begin{abstract}
Current draft annotations for sequenced conifer genomes are preliminary and limited, but provide opportunities for further structural and functional analysis. We attempted to improve the existing genome annotations by marking 5'-UTRs in the four conifer species Pinus taeda, Picea glauca, Picea abies and Larix sibirica. Prediction of transcription start sites (TSS) was performed on the promoter sequences of genes with RNA or protein support using TSS prediction program TSSPlant. The distribution of 5'-UTR lengths from the annotations of several model plants was used to select the best prediction per gene. Frequency of TATA(A/T)A(A/T) motif in the predicted TSS-centered promoter regions showed a pronounced peak around $60 \mathrm{bp}$ upstream of TSS.
\end{abstract} biology

Keywords - 5'-UTR, plant promoters, conifer genome, $\mathrm{GC}_{3}$

\section{Introduction}

Conifers are an ancient group of plants represented by more than 600 species that play major role in boreal forest ecosystems. Due to the enormous size and highly repetitive nature deciphering conifer genomes takes more time and effort than many other plant species. In the last years several mega-genomes of conifer species were sequenced and assembled to the draft state. Their current annotations are preliminary and limited, but provide opportunities for structural and functional analysis. Understanding of regulatory relationships between genome elements requires information on promoter sequences, which are usually located directly upstream or at the $5^{\prime}$ end of the transcription start site (TSS). Here we present our attempts to improve the existing genome annotations by predicting TSSs and marking 5'-UTRs in the four relatively recently published species Pinus taeda, Picea glauca, Picea abies, and Larix sibirica.

\section{Materials and Methods}

\section{A. Genome Assemblies and Annotations}

The following genome assemblies and annotations of four conifer species were used: Pita v2_01 for Pinus taeda $[1,2]$, PG29 v3.0 for Picea glauca [3, 4], Pabies v1.0 for Picea abies [5], and genome assembly under GenBank accession NWUY0000000000 (BioProject PRJNA393226) together with the unpublished draft annotation data for Larix sibirica [6].

\section{B. Gene Filtering}

To filter out possible pseudogenes and putative predicted coding sequences that do not present functional genes all gene models retrieved from genomic annotations were aligned against the database of RNA-seq data, including ESTs and TSAs, of a corresponding species using hisat 2 alignment program. To additionally verify selected gene models we aligned corresponding protein products to Refseq plant protein sequences using blastp program.

\section{Transcription Start Site (TSS) Prediction}

Prediction of putative TSSs was performed in the promoter sequences of selected genes, which were defined as regions of -1000 and +250 bp around the start codon, using TSSPlant program [7] and selection of the best prediction based on the distribution of 5'-UTR lengths from the annotations of several model plants.

\section{D. $G C_{3}, C G$-skew and Frequency of TATA motif}

Nucleotide frequency analysis of promotors was performed on TSS-centered sequences $(-1000$ and $+200 \mathrm{bp}$ around TSS). Frequencies of CA and TATA motifs were calculated with a sliding window (width $=40 \mathrm{bp}$, increment step $=10 \mathrm{bp}$ ) using stringr package for R. CG-skew of a given sequence was defined as a proportion $(\mathrm{C}-\mathrm{G}) /(\mathrm{C}+\mathrm{G})$ and 
calculated with sliding window width of $50 \mathrm{bp}$ and window increment step of $10 \mathrm{bp}$ along the promoter sequence. $\mathrm{GC}_{3}$ was calculated using gene sequences with removed introns (only joined exons were used) and seqinr package for $\mathrm{R}$.

\section{Results}

Aligning to RNA-seq, ESTs, and Refseq protein data allowed to retrieve 9260 evidence supported gene models for P. taeda, 16853 for P. glauca, 7587 for P. abies, and 23077 for L. sibirica (Table I). For these genes (both TATA and TATA-less) TSSs were predicted using TSSPlant algorithm. Frequency of TATA(A/T)A(A/T) motif in the predicted TSScentered promoter regions showed pronounced peak around $60 \mathrm{bp}$ upstream of TSS (Fig. 1A). All four species have similar $\mathrm{GC}_{3}$ distribution with the average around 0.3 and $\mathrm{GC}_{3}$ gradient that gradually decreases after $250 \mathrm{bp}$ from the start of coding sequence (Fig. 1B). In contrast to what was observed in A. thaliana, O. sativa, and some other plant species, CG-skew in four conifer species exhibited distinct decline right before the TSS (Fig. 1C).

TABLE I.

SUMMARY OF ASSEMBLIES AND ANNOTATIONS

\begin{tabular}{|l|l|l|l|l|}
\hline \multicolumn{1}{|c|}{ Assembly } & $\begin{array}{c}\text { Assembly } \\
\text { N50 }\end{array}$ & $\begin{array}{c}\text { Sum } \\
\text { length, } \\
\text { Gb }\end{array}$ & $\begin{array}{c}\text { Number } \\
\text { of genes }\end{array}$ & $\begin{array}{c}\text { RNA/Refseq } \\
\text { supported } \\
\text { genes }\end{array}$ \\
\hline P. taeda v2_01 & 100218 & 20.43 & 36732 & 9260 \\
\hline P. abies v1.0 & 7747 & 9.9 & 58587 & 10434 \\
\hline PG29 v3 & 34405 & 20.8 & 103694 & 16839 \\
\hline L. sibirica & 3098 & 5.6 & 50163 & 23077 \\
\hline
\end{tabular}
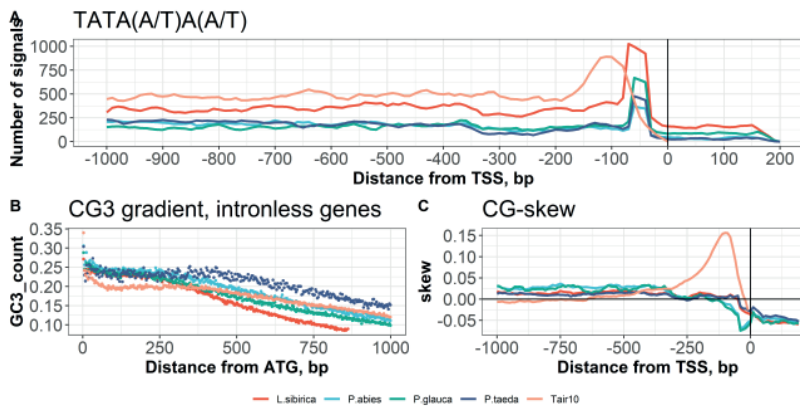

Fig. 1. Frequency of $\operatorname{TATA}(\mathrm{A} / \mathrm{T}) \mathrm{A}(\mathrm{A} / \mathrm{T})$ motif in the TSS-centered promoter region $(\mathrm{A}), \mathrm{GC} 3$ gradient of coding sequences $(\mathrm{B})$, and CG-skew around TSS (C).

\section{Acknowledgments}

We thank Dr. N. V. Oreshkova and Dr. S. I. Feranchuk for help with sequencing and draft annotation of the Larix sibirica genome, respectively.

\section{References}

[1] D. B. Neale, et al. "Decoding the massive genome of loblolly pine using haploid DNA and novel assembly strategies", Genome biology 15 no. 3 , March 2014, p. R59.

[2] A. V. Zimin, et al. "An improved assembly of the loblolly pine megagenome using long-read single-molecule sequencing", GigaScience 6, no. 1 January 2017 , p. giw016.

[3] I. Birol, et al. "Assembling the $20 \mathrm{~Gb}$ white spruce (Picea glauca) genome from whole-genome shotgun sequencing data", Bioinformatics 29, no. 12, Jun 2013, pp. 1492-1497.

[4] R. L. Warren, et al. "Improved white spruce (Picea glauca) genome assemblies and annotation of large gene families of conifer terpenoid and phenolic defense metabolism", The Plant Journal 83, no. 2, May 2015, pp. 189-212.

[5] B. Nystedt, et al. "The Norway spruce genome sequence and conifer genome evolution", Nature 497, no. 7451, May 2013, pp. 579-584.

[6] D. A. Kuzmin, et al. "Stepwise large genome assembly approach: a case of Siberian larch (Larix sibirica Ledeb)", BMC bioinformatics 20.1, Feb 2019, 37.

[7] I. A. Shahmuradov, R. K. Umarov, V. V. Solovyev, "TSSPlant: a new tool for prediction of plant Pol II promoters", Nucleic acids research, 5;45(8), May 2017, pp. e65-e65. 